\title{
Design of a Prototype System Operant in Lunar Environment
}

\author{
Rita Ambu* - Andrea Manuello Bertetto - Costantino Falchi \\ University of Cagliari, Department of Mechanical, Chemical and Materials Engineering, Italy
}

In this paper, the design of a prototype system developed for a rover intended for the removal and transport of rocks on lunar soil is reported. The part of the rover dedicated to some of the main tasks, i.e. the lifting of objects and moving on rugged terrain, while controlling of the balance of the vehicle, is considered. These tasks are accomplished through the mechanical components assembled in a column connected to wheels. The study has been conducted with the aim of obtaining a simple and lightweight structure satisfying the requirements necessary to operate on the lunar soil.

After a description of the architecture of the rover, the layout of the components of the column is detailed. A compliant, spiral-spring wheel is proposed to complete the mobility system. The primary components of the column are then structurally assessed by means of FEM numerical simulations. A numerical model of the wheel has also been implemented, in order to define in detail the wheel geometry and performance.

The proposed layout could be promising for lunar applications since it has a configuration suitable for the specific characteristics of the environment it has to operate.

Keywords: design, working operation, lunar environment, column, wheel, FEM simulation

\section{INTRODUCTION}

There has long been great interest in the exploration of the moon. Different missions have been carried out over the years, including the Apollo missions [1], which enabled the collection of a large amount of data, including the physical and chemical characteristics of the environment and the configuration of the soil [2].

The information collected during the lunar missions has also enabled the possibility of establishing installations with human presence on the planet for the potential advantages that this may offer, including space tourism, which has been demonstrated to be an economically viable activity [3]. Several types of structures have been proposed for lunar outposts, ranging from simple inflatable structures to more advanced projects with complex buildings and supporting infrastructure [4] to [6].

However, the installation of a facility on the moon will require extensive operations for proper site preparation, including terrain excavation and transportation of rocks; furthermore, complementary mining operations can be advantageous since construction materials would be prepared from local materials and life support materials such as oxygen and water may be provided by indigenous resources [7].

The essential tasks of the devices required for working operations are those typical of construction machines, but different design solutions are necessary to create mechanical devices that can efficiently operate on the lunar soil.
Generally, the design of the components of a lunar vehicle has to take into account the peculiar characteristics of the fine and dry soil called lunar regolith, as well as those of the environment in which it will have to operate, e.g. temperature and radiation effects [8].

Previous lunar rovers used in exploration missions were assigned to transport crew, exploring the terrain and collecting the material samples. These vehicles, the Lunokhod and the Apollo Lunar Roving Vehicle (LRV) [9], were primarily designed to move in dusty environments.

In the design of these vehicles, primary attention was given to the mechanical components that directly interact with the soil; in the development of rovers assigned to working missions, in addition to mobility, characteristics such as the load-bearing and carrying capability in a hostile environment also have to be accurately considered, while simultaneously keeping the structure simple and versatile.

In recent years, several robot prototypes have been developed specifically for lunar excavation [10] to [12] and in situ resource utilization (ISRU) [13] and [14], which have varied widely in weight and tooling configuration. Bucket-wheel excavators have been shown to produce low-resistance forces when taking only small portions of regolith at a time; however, by repeatedly taking a large number of these small portions, high production is maintained.

This paper deals with the research relative to the vehicles assigned to working missions on the lunar soil. In particular, it reports the design of a basic part of a rover, under development, conceived 
to clear rocks from areas allocated to buildings or infrastructure. The device discussed in this paper is conceived as an auxiliary device to work on the lunar soil together with other machines; therefore, a direct comparison with other proposed design solutions of working devices, such those in references from 10 to 14 , is difficult or inappropriate.

The primary job of this rover is to grab rocks and move them, and comprises a gripping mechanism and four columns on which the wheels are assembled. In this paper, the components of the column have been considered, with particular attention to the lifting mechanism. The architecture of the wheel has also been developed.

This study has been conducted via an analysis of a suitable arrangement and the choice of the materials of the components to obtain a structure to optimally operate in the lunar environment, while simultaneously minimizing size and mass. Parametric computer-aided design (CAD) modelling was used to determine the geometry of the components while the structural performance of the most significant parts under extreme loading conditions was assessed by means of finite element modelling (FEM) numerical simulations. Analogous methods were employed to define the characteristics and the performance of the wheel. A prototype of the column and the wheel was finally manufactured to verify the proposed configuration.

The main aim of this paper is to propose and discuss a feasible configuration of a subsystem of a lunar rover, establishing suitable geometry for the components and quantitatively verifying with numerical simulations the stress level and loadbearing capability. Since the vehicle project is an extensive study, the results reported here are focused on a particular part of the overall design.

\section{OVERVIEW OF THE ROVER}

The design of the rover was aimed to obtain a functional structure able to accomplish the working tasks described in the introduction, while simultaneously satisfying general requirements.

A primary requirement is relative to the vehicle mobility since the design should satisfy specific geographical conditions, such as the ability to cross obstacles. Furthermore, it has to have sufficient endurance for working operations, including reliability and robustness in the environment. Thirdly, overall system efficiency requirements, such as low weight and compact size, must be satisfied.
The vehicle specifications are reported in Table 1, which summarizes the main geometric and performance characteristics of the vehicle.

Table 1. Vehicle specifications

\begin{tabular}{lc} 
Length [mm] & 1855 \\
\hline Width [mm] & 918 \\
\hline High $[\mathrm{mm}]$ & 1627 \\
\hline Mass [kg] & 200 \\
\hline Avg. speed (in plane) [m/s] & 0.5 \\
\hline Max. speed (in plane) [m/s] & 1 \\
\hline Maximum terrain slope $\left[{ }^{\circ}\right]$ & 30 \\
\hline Number of wheels $[-]$ & 8 \\
\hline
\end{tabular}

The rover is designed to grasp and lift objects with weighs up to $800 \mathrm{~N}$, corresponding to a mass of about $500 \mathrm{~kg}$ in the lunar gravity.

A schematic diagram of the vehicle with the main components is reported in Fig. 1.

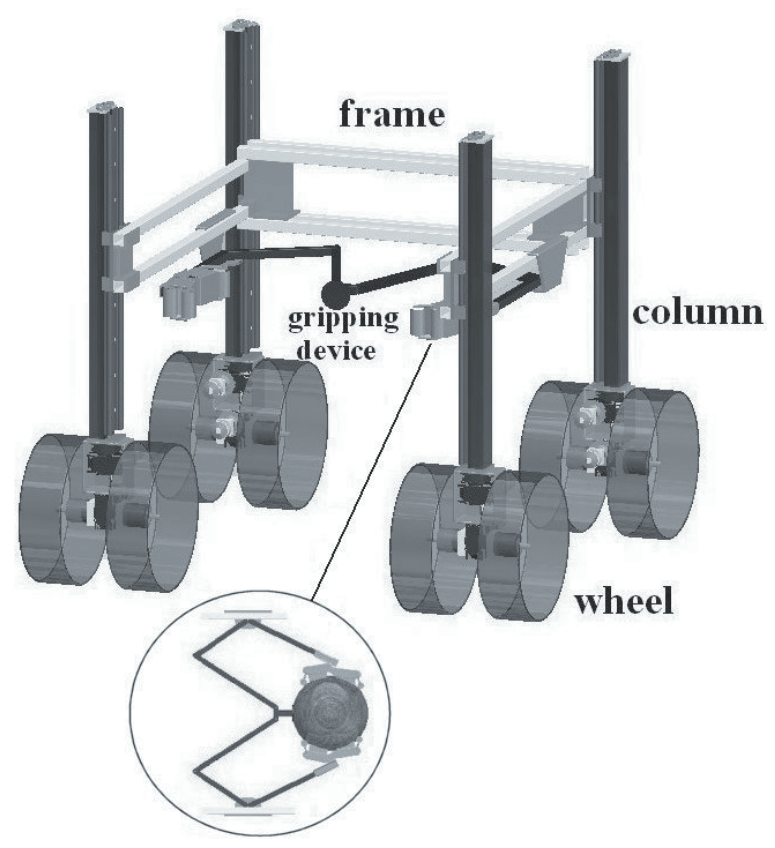

Fig. 1. The architecture of the rover

It comprises a rigid frame to which the gripping mechanism of the load and four columns supported on the wheels are connected. The columns transmit the load to the frame by means of slides assembled to the frame. The gripping system [15], whose detail in a grasping position is also reported in the lower part of Fig. 1, is a plane-articulated mechanism with three degrees of freedom. The layout this mechanism allows it to grasp solid objects of different shapes, such as the numerous rocks on the lunar surface. 
This architecture makes the rover easily transportable and, possibly, adapted to reuse in the lunar base for the transportation of various objects.

\section{DESCRIPTION OF THE LAYOUT OF THE PROTOTYPE SYSTEM}

The columns of the rover have been designed to accomplish different essential tasks for correct operations.

The requirements that the proposed configuration will have to satisfy are to propel the vehicle, lift the load, and control the balance in relation to the contour of the soil.

In this paragraph, the geometric configuration of the components is described, while the structural analysis is reported later. The paper is focused on the geometrical configuration and structural performance, while a future active control to steer the vehicle trim is foreseen. However, the fundamental issue of control strategies is behind the aim of this paper and is not discussed here.

The prototype column of the rover is shown in Fig. 2; at the right side, a detail of the lower part is also reported, showing the assemblage of the wheels and the motors.

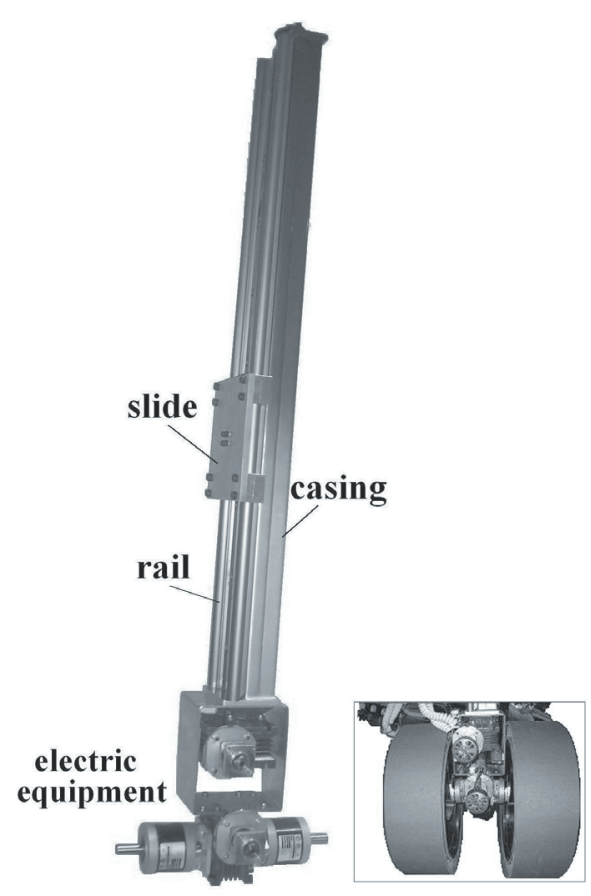

Fig. 2. The column of the rover

The lifting mechanism, which controls the shift of the load and enables the vehicle to remain on course, comprises a slide joined to two linear guides with a low coefficient of friction, a precision coupling between a lead screw and nut driven by an electric motor and a particular device, which will be later described in detail, designed for the correct operating of the screw and nut mechanism. The electric motor used for the lifting mechanism is a high efficiency brushless motor with an electrical power of about one kilowatt and a mass less than one kilogram. The shaft of the electric motor is connected to a series connection of two reduction gears, an epicyclical reduction gear and a worm screw gear, with an overall speed ratio of 30 .

This layout allows choosing the position of the frame of the rover according to the size and geometry of the rock. Furthermore, since each column can be driven independently, the vehicle can cover rugged terrain and cross obstacles as large as the diameter of its wheels maintaining a proper balance, thus restricting the likelihood of overturning during motion on rough terrain.

The correct operating of the screw and nut coupling under load is assigned to a decoupling joint that is inserted between the screw and nut mechanism and the slide.

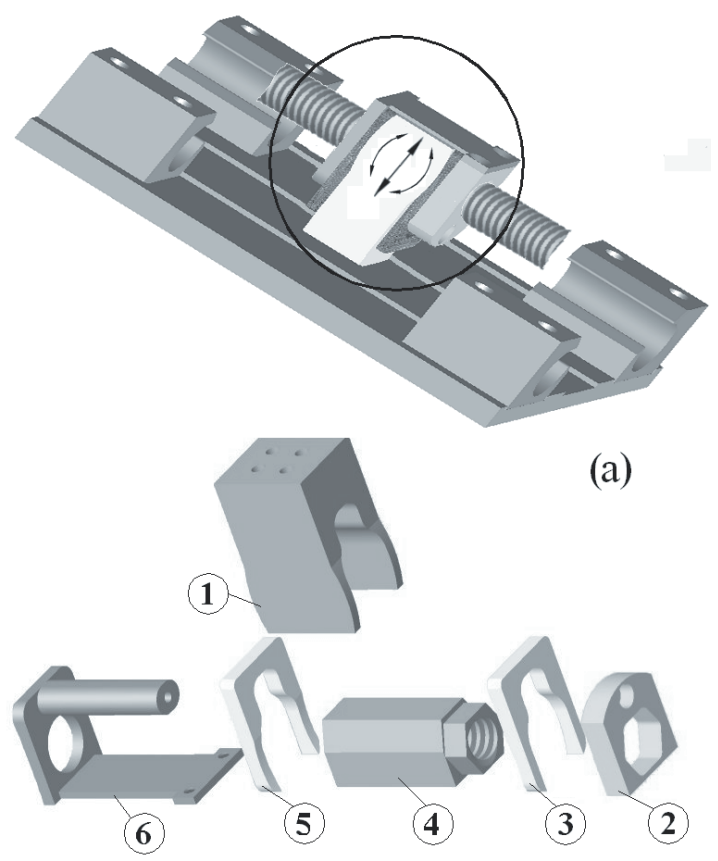

(b)

Fig. 3. A detail of the column; a) the plate and the screw and nut mechanism, and $b$ ) the components of the decoupling device and the nut

Fig. 3a shows the portion of the column with the slide and its connection to the screw and nut 
mechanism; the area that includes the joint has been highlighted in the figure. The components of this device and the nut are assembled in a compact apparatus, since the size and shape of each part were chosen so as to minimize the overall volume.

The exploded view shown in Fig. $3 b$ shows the components of the assembly between the joint and the nut.

In particular, with regards to Fig. $3 b$, the joint (1) is rigidly connected to the slide, and it is simply placed on the flange of the nut (2) interposing a meniscus (3) between the two parts to reduce the friction between the surfaces. These components are finally assembled, introducing another similar meniscus (5) at the opposite end of the joint, and a proper counterpart (6) fastened to the flange of the nut by means of a screw.

The joint, whose geometry has been accurately optimized [16], was designed for transmitting to the lead screw only the normal component of the load, simultaneously transferring the other components to the load-bearing structure. In fact, the geometry and the assembly conditions give this component the capability of both rotating about an axis perpendicularly to the axis of the screw and of sliding in a direction orthogonal to the same axis, also compensating the potential misalignment between the axis of the screw and the axes of the rails fixed to the column.

The arrangement of the components of the lower part of the column was intended to be a system in which the transmission, suspension and motorization mechanisms are integrated into a compact apparatus. The motion of the rover and the selection of the trajectory are obtained by means of a differential traction that controls a pair of wheels driven by an electric motor. The electric motor chosen for the motion of the rover has specifications analogous to that used for the lifting mechanism, satisfying the mobility requirements, as well as those of low mass and size. The gears that drive the wheels have an overall speed ratio of 960 between the motor shaft and the wheels, for an efficiency ratio of about 0.30 . This speed ratio allows it to have an irreversible system by means of a kinematic chain with a worm gear and an epicyclical gear.

The design of the architecture of the wheel was conducted starting from a preliminary analysis of the different configurations used in previous lunar missions. These spread from the wheel made of a rigid structure with a wire carcass connected by spokes used in the Lunokhod vehicle to a flexible wheel with a wire mesh carcass and a stiff inner frame employed in the LRV [17]. Apart from the two cited, the other wheel used on the moon soil, which operated on the mobile equipment transporter (MET), was made of rubber. Following the general requirements derived from the previous practice, the wheel architecture also was developed taking into account the specific requirements of the proposed vehicle and a more recent study on non-pneumatic wheels [18] whose design also benefits of the use of advanced composite materials instead of the traditional ones.

The conceived wheel is shown in Fig. 4.

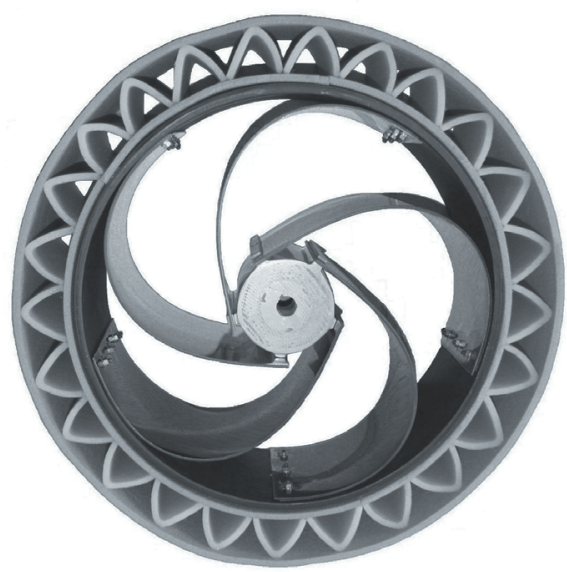

Fig. 4. The wheel

The essential components are a central hub, a series of spiral springs, geometrically shaped as bitangent semicircles, and a tread which is composed of non-rigid triangular elements with straight sides. The triangular shape was the only geometry considered and was chosen for its efficiency in transmitting the driving and braking torque. The spokes are fixed to the hub and to a rigid rim.

This configuration allows the wheel to perform different tasks, such as those of elastic and dissipative suspension and the reduction of the effects of the local asperities of the soil on the vehicle. The spiral springs act as elastic elements in order to provide both load bearing and shock absorption capabilities.

The triangular elements of the tread undergo local deformation when the rover crosses an asperity of the rugged terrain, thereby avoiding transferring possible stresses to the structure.

\section{NUMERICAL ASSESSMENT OF THE PROPOSED LAYOUT}

\subsection{The Lifting Mechanism}

The components directly involved in the lifting of the weights and the balance of the vehicle were considered in order to evaluate the performance of the column. 
In particular, the lead screw and nut coupling and the connection of this mechanism to the slide, which was accomplished by means of the decoupling joint, have been analysed in detail.

A standard Acme thread profile was selected for the screw and the nut with a nominal screw diameter of $15.5 \mathrm{~mm}$. The nut was designed in two parts, both depicted in Fig. 3b, consisting of a prism-shaped part internally threaded, labelled as (4) in the figure, and a plane element, labelled as (2), which serves as the flange. The use of a nut made into two parts is also advantageous for manufacturing purposes.

The lead screw and nut coupling was structurally verified by means of finite element analysis. The parametric CAD modelling used for the design of each component, among the many advantages, also allowed to perform the structural validation of the real geometries. In fact, the effects of the lead angle and the helix of the threaded profile, neglected in axisymmetric finite element models, can be taken into account.

The finite element model of the two parts, implemented in a FEM commercial software (ANSYS), was obtained via 142,133 3D-brick elements, while contact elements were introduced at the interface between the threaded surfaces of the screw and the nut.

Fig. 5 shows the FE model obtained.

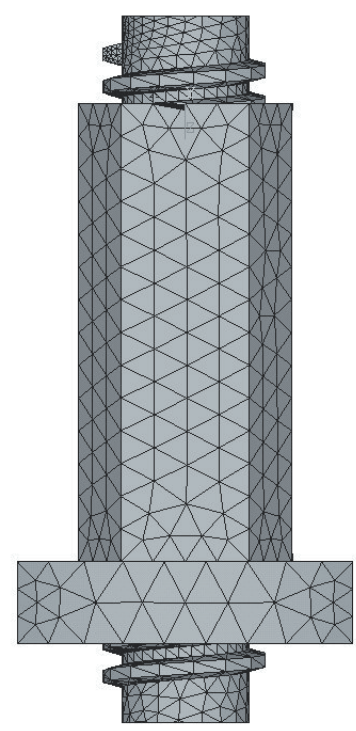

Fig. 5. FE model of the screw and nut mechanism

A composite material with a thermoplastic matrix (Nylon6/6) reinforced with 30\% carbon fibres and 5\% PTFE was chosen $(E=14500 \mathrm{MPa}, v=0.3)$ for the nut, and it was coupled with a titanium alloy $(E=111000$
$\mathrm{MPa}, v=0.3$ ) lead screw. The numerical simulations were relative to an extremely heavy limit condition. In fact, during the working operations on the lunar soil, an unexpected balance condition of the vehicle can occur when it can accidentally be sustained only on two of the four columns. If this happens while the rover is loaded at its upper limit, the two operating columns are forced to carry the maximum load.

The stresses on the mechanism in this condition were evaluated, giving particular attention to the analysis of the nut, since it is the weakest component of the coupling. As for the lead screw, the maximum value of the Von Mises stress obtained was $292 \mathrm{MPa}$, about the $30 \%$ of the ultimate stress for the chosen material.

Fig. 6 reports an iso-colour representation of the Von Mises stress distribution in the nut, expressed in MPa.

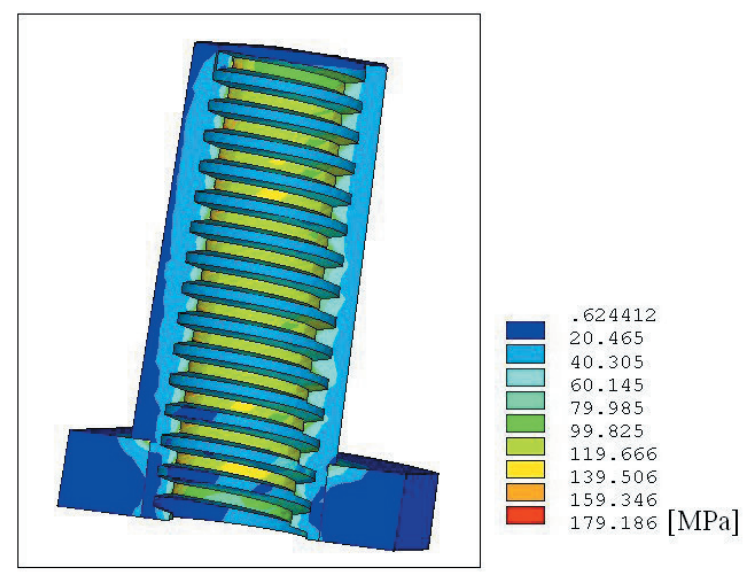

Fig. 6. Von Mises stress distribution in the nut

The component exhibits the presence of stress concentrations along with the edges of the threaded surfaces, especially in correspondence to the threads next to the outer side, but with a maximum stress that is under the ultimate stress value (221 MPa) of the material.

To further validate the proposed geometry, the distribution of the axial load on the threads of the nut was investigated. In the design of screw and nut transmission mechanisms, it is usually assumed that the axial load is equally distributed on the threads. However, the load distribution on the threads can be affected by the nut contour, size, stiffness, and other factors [19]. For this purpose, a parameter, i.e. the ratio of flank load in percentage (RFL\%) that represents the load carried by each thread, was calculated. 


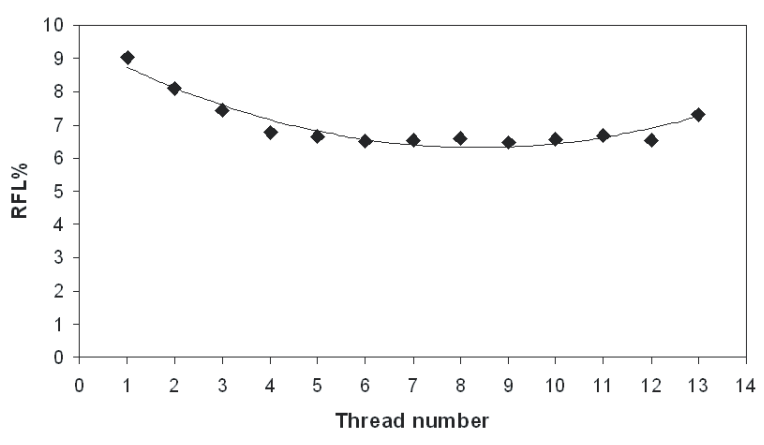

Fig. 7. Axial load distribution on the threads of the nut

Fig. 7 depicts a diagram that summarizes the results obtained. The diagram depicts on the vertical axis the parameter RFL\%, while on the horizontal axis the thread number starting from the nut fixed surface, corresponding to the flange surface, is reported. A trend line has also been added to the plot.

The load distribution exhibits higher values at the outer engaged threads; however, the maximum difference between the higher and lower value is rather limited $(2.6 \%)$, thus assessing the proposed geometry.

As described in the previous paragraph, the screw and nut mechanism was completed with a decoupling device. The functional parameters of this device were first evaluated. It was accomplished by a mechanical variation analysis of the assembled device evaluated at the limit positions by means of a tolerance analysis software. In particular, since the joint was conceived to allow linear and angular adjustments, the maximum linear displacement and the maximum angle of rotation were estimated. As for the linear displacement, a value of about $2.5 \mathrm{~mm}$ was obtained, while a maximum angle of $4^{\circ}$ was estimated. These values, even if limited, can be considered satisfactory, since the adjustment of the position of the slide will mainly occur via a combined variation of the two parameters.

To assess the structural performance of the decoupling device, it was necessary to take into account the interaction of the joint with the meniscus interposed between its surface and the nut, thus considering in the numerical simulations the joint and the meniscus as a coupled pair. The simulations were relative to the same extreme operating condition previously described for the screw and nut coupling, where only two columns are supposed to bear the overall load.

The finite element model was made with 32,372 3D-brick elements, while contact elements were introduced at the interface between the joint and the meniscus. The symmetry of the geometry was taken into account by modelling only one half of each part and introducing symmetry boundary conditions. The bottom surface of the meniscus was fixed while the load was applied at the end surface of the joint.

An aluminium alloy $(E=69000 \mathrm{MPa}, v=0.3)$ was the material chosen for the joint, while a thermoplastic polyamide $(E=2010 \mathrm{MPa}, v=0.49)$ was selected for the meniscus.

Fig. 8 shows the FE model of the coupled components.

In the analysis of the results, particular attention was given to the meniscus, regularly allocated to assist the sliding of the joint, which is the weakest component between the two parts and prone to wear.

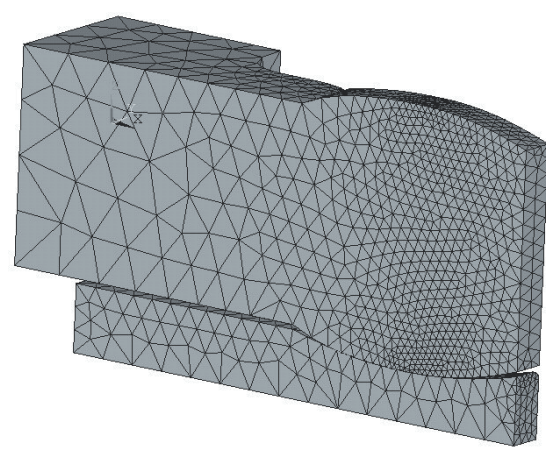

Fig. 8. FE model of the joint and meniscus

Fig. 9 shows an iso-colour representation of the Von Mises stress distribution relative to this component where the surface in contact with the joint is visible.

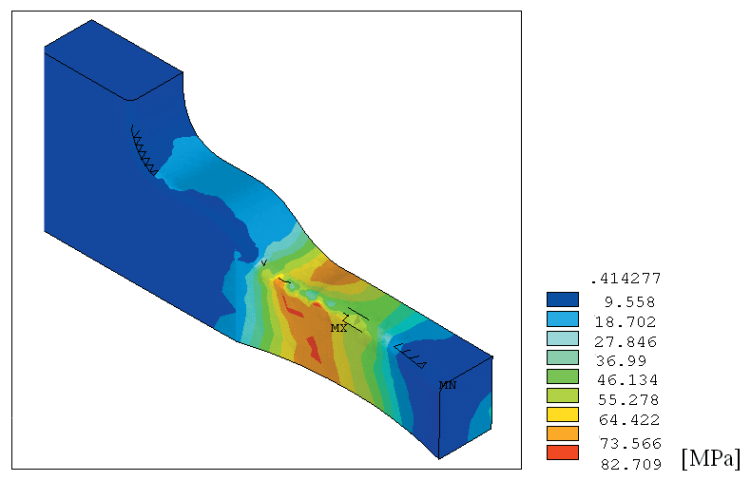

Fig. 9. Von Mises stress distribution in the meniscus

In Fig. 9, the local effect on the stress distribution of the peculiar loading condition can be observed; however, higher values are limited within a restricted area and do not exceed the maximum admissible value (104 MPa) for the material chosen. 


\subsection{The Wheel}

The aim of the analysis was to determine the wheel characteristics satisfying the requirements with the minimum cost in terms of mass. The wheel configuration depends on different parameters. The values of some of them, such as the wheel external diameter and the wheel width, are dictated by the system requirements, while the values of other parameters can be chosen and varied appropriately. These last parameters include the number of springs, their curvature and wall thickness, as well as the materials of the wheel components, i.e. the tread, the hub and the springs. Different sets of values of these parameters have been considered, combining parametric CAD modelling and FEM analysis.

The approach considered consists of the analysis of different configurations obtained by changing the values of the input parameters such as the geometry and the thickness of the spokes. The outputs of each FEM analysis considered are the wheel deformation and the internal stress. This procedure considered discrete values of the geometric parameters of the components and some materials compatible with the lunar environment. However, this procedure does not exhaust all prospective configurations. The result of the activity has been to precisely define a wheel configuration compliant with the system requirements.



Fig. 10. FE model of the wheel

The wheel width is $150 \mathrm{~mm}$ and the outer diameter is $500 \mathrm{~mm}$ while the inner diameter of the tread has a value of $400 \mathrm{~mm}$. The spokes, which consist of 5 radial elements, are curved surfaces with a radius varying between 119 and $126 \mathrm{~mm}$. The hub diameter is $100 \mathrm{~mm}$.
The FE model of the wheel, reported in Fig. 10, was made with 39,812 3D-brick elements. A rigid plate was introduced in order to simulate the ground. Contact elements were inserted between the plate and the tread to hinder penetration between the wheel and the plate.

A glass fibre reinforced epoxy composite material $(E=11900 \mathrm{MPa}, v=0.217)$ was chosen for the spiral spokes while the tread was made of rubber $(E=5$ $\mathrm{MPa}, v=0.48)$. In addition, an aluminium hub was used.

A vertical load was applied on the wheel hub in order to simulate the load transfer on the wheel, while the plate was fixed.

Since the rover is a low-speed vehicle, as reported in Table 1, only a static analysis has been examined. Suspensions allow effective behaviour at the interface with the ground, in order to have a valid exchange of forces with it, also in view of foreseen control strategies of trim and traction. Furthermore, a global analysis is reported in this study, since the entire system and the wheel subsystem are non-linear. Distinguishing the contribution of the different parts and taking into account each separately is considered to be less significant for the evaluation of the system. In any case, it is certainly important to evaluate the influence of each part on the global behaviour, but it has been not been considered here.

Fig. 11 shows the vertical displacement obtained as a function of the vertical applied load, which allows to evaluate the trend of the wheel stiffness, i.e. the resistance of the wheel to the deformation when a vertical load is applied.

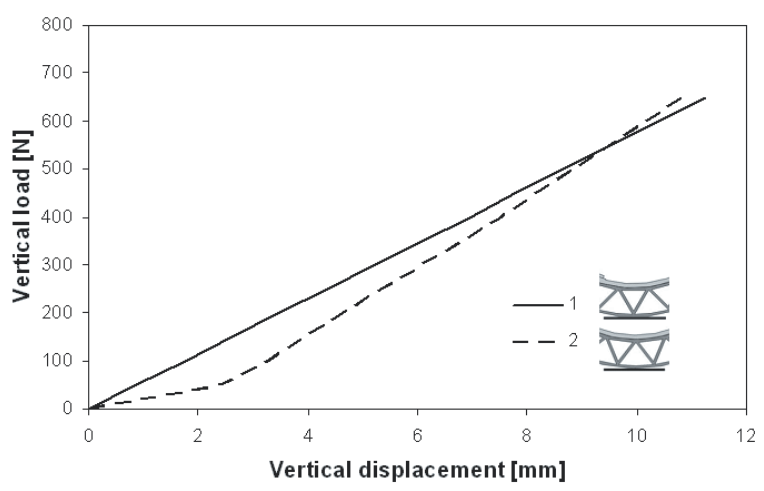

Fig. 11. The force-displacement curve of the wheel

Two different positions that repeatedly alternate during the motion of the vehicle were considered. The first, corresponding to Curve 1 (solid line) in the figure, was relative to a vertex of a triangular element of the tread positioned on the vertical symmetry axis 
of the wheel, while Curve 2 (dashed line) corresponds to the vertical axis of the wheel positioned between two vertices of a triangular element.

Curve 1 shows a linear trend in the range considered, while Curve 2 has an analogous tendency except for lower values of the vertical applied load. The difference between the values of the vertical displacements relative to the two positions decreases as the applied load increases; in particular, around the design load $(365 \mathrm{~N})$, the difference is less than $1 \mathrm{~mm}$.

A change of stiffness during rotation should be troubling regarding the transmission of vibrations to the system. However, it can be advantageous to achieve a greater efficiency in the transmission of the drive or braking torque to the ground on soft terrain, due to an effect similar to that of a groove of the tread.

The implemented numerical model also enables evaluating basic parameters, such as the wheel-ground contact area and the contact pressure. The contact area under the nominal load can be estimated to be 5985 $\mathrm{mm}^{2}$, yielding an average pressure of $64.89 \mathrm{kPa}$ on the ground.

Finally, the stress relative to the maximum load acting on the wheel, corresponding to the column sustained on only one wheel of the couple, was evaluated.

Fig. 12 depicts an iso-colour representation of the Von Mises stress distribution obtained.

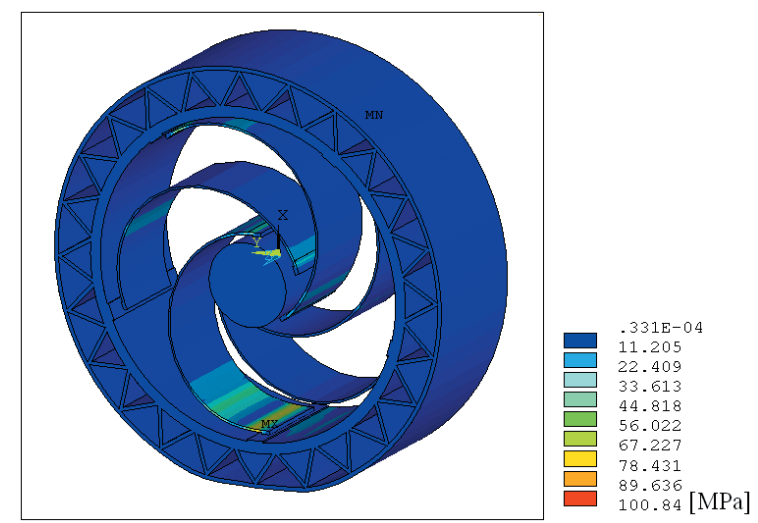

Fig. 12. Von Mises stress distribution in the wheel

The maximum value of the equivalent stress is under the limit stress value and occurs in a small zone at the interface between the rim and a spoke, suggesting further improvement of the joints between these components of the wheel.

\section{CONCLUSIONS}

In this paper, a prototype system of a column and wheel, essential components of a rover allocated to working tasks on the lunar soil, is proposed and assessed via numerical simulations.

The resulting structure of the vehicle is straightforward and lightweight, which offers many advantages both for shipping and during operation on the lunar soil.

It can be adjusted to function on rough terrain thanks to the ability to traverse obstacles by longitudinally changing the position of each pair of wheels. In addition, severe loading conditions can be sustained by the main parts, as shown via the numerical simulations.

Future work will be concerned with the experimental verification of the prototype rover in an environment simulating the characteristics of the lunar soil.

\section{REFERENCES}

[1] Harland, D.M. (2008). Exploring the moon: the Apollo Expeditions. Springer-Praxis, Berlin, DOI:10.1007/978-0-387-73997-7.

[2] Heiken, G.H., Vaniman, D.T., French, B.M. (1991). Lunar Sourcebook, A User's Guide to the Moon, Cambridge University Press, Cambridge.

[3] Collins, P. (2006). The economic benefits of space tourism. Journal of the British Interplanetary Society, vol. 59, p. 400-410.

[4] Grandl, W. (2007). Lunar base 2015 stage 1 preliminary design study. Acta Astronautica, vol. 60, no. 4-7, p. 554-560, DOI:10.1016/j.actaastro.2006.09.031.

[5] Jones, T.D. (2007). Homesteading the Moon. Aerospace America, vol. 45, no. 4, p. 12-15.

[6] Benaroya, H., Bernold, L. (2008). Engineering of lunar bases. Acta Astronautica, vol. 62, no. 4-5, p. 277-299, DOI:10.1016/j.actaastro.2007.05.001.

[7] Chamberlain, P.G., Taylor, L.A., Podnieks, E.R., Miller, R.J. (1993). A review of possible mining applications in space. Lewis J.S., Matthews, M.S., Guerrieri, M.L. (eds.). Resources of Near-Earth Space. The University of Arizona Press, Tucson, p. 51-68.

[8] Pirich, R., Weir, J., Leyble, D., Chu, S., DiGiuseppe, M. (2010). Effects of the lunar environment on space vehicle surfaces. Proceedings of Long Island Systems, Applications and Technology Conference, Farmingdale, p. 1-6.

[9] Young, A. (2007). Lunar and planetary rovers: the wheels of Apollo and the quest for Mars. SpringerVerlag, Berlin.

[10] Skonieczny, K., Moreland, S.J., Wettergreen, D.S., Whittaker, W.L. (2011). Advantageous bucket-wheel configuration for lightweight planetary excavators. 
Proceedings of 17th International Conference of the International Society for Terrain Vehicle Systems, Blacksburg.

[11] Johnson, L.L., King, R.H. (2010). Measurement of force to excavate extraterrestrial regolith with a small bucket-wheel device. Journal of Terramechanics, vol. 47, no. 2, p. 87-95, DOI:10.1016/j.jterra.2009.08.002.

[12] Nasa (2013). Engineers building hard-working mining robot, from http://www.nasa.gov/topics/technology/ features/RASSOR.html, accessed on 2013-14-05.

[13] Bartlett, P.W., Wettergreen, D., Whittaker, W. (2008). Design of the scarab rover for mobility and drilling in the lunar cold traps. Proceedings of International Symposium on Artificial Intelligence, Robotics and automation in Space, Hollywood.

[14] Astrobotics (2013). Polaris, from http://astrobotic.net/ rovers/polaris, accessed on 2013-18-06.

[15] Carbone, G., Falchi, C., Manuello Bertetto, A., Ceccarelli, M. (2012). Simulation of a gripping device for obstacle removing on lunar soil. Proceedings of $21^{\text {st }}$
International Workshop on Robotics in Alpe-AdriaDanube Region, Naples.

[16] Ambu, R., Falchi, C., Manuello Bertetto, A. (2010). A lunar rover leg: optimal design of a decoupling joint. International Journal of Mechanics and Control, vol. 11, no. 1, p. 45-50.

[17] Asnani, V., Delap, D., Creager, C. (2009). The development of wheels for the lunar roving vehicle. Journal of Terramechanics, vol.46, no.3, p. 89-103, DOI:10.1016/j.jterra.2009.02.005.

[18] Rhyne, T.B., Cron, S.M. (2006). Development of a non-pneumatic tire. Tire Science and Technology, vol. 34, p. 150-169, DOI:10.2346/1.2345642.

[19] Sun, Y., Zhou, X., Wei, L., Wang, W. (2009). Development of a new type of transmission screw nut with high efficiency and heavy duty characteristics. Proceedings of the Institution of Mechanical Engineers, Part C: Journal of Mechanical Engineering Science, vol. 223, no. 5, p. 1181-1189, DOI:10.1243/09544062JMES1332. 